\title{
Integrierte Versorgung: Aufbruch in die Zukunft
}

\section{Leander Muheim, im Namen der Arbeitsgruppe Managed-Care-Workshop}

Dr. med., stv. medizinischer Leiter medix zürich

Vom 7. bis 9. Dezember 2017 trafen sich führende Vertreter aus Ärztenetzen, Krankenversicherungen und Gesundheitspolitik in Scuol zum 20. Managed-CareWorkshop. Das Treffen stand ganz im Zeichen des anstehenden Systemwandels im Bereich der integrierten ambulanten Versorgung.

\section{Herausforderungen im Rahmen der weiteren Entwicklung von Managed Care}

Die Situation aus ärztlicher Sicht

Eingangs erläuterte Felix Huber von mediX die Situation aus ärztlicher Sicht: Branchenfremde Akteure würden mit Hoffnung auf gute Renditen breit in den Gesundheitsmarkt investieren und für eine weitere Mengenausweitung sorgen. Die Managed-Care-Bewegung (MC) habe bislang nicht mit genug Dynamik auf diese Entwicklung reagiert. Kritisch beurteilte er die- jenigen Krankenkassen, welche seiner Meinung nach weiterhin alternative Versicherungsprodukte ohne integrierten Versorgungsansatz zu Dumpingpreisen auf den Markt werfen würden und damit das echte Hausarztmodell unterbieten. Es könne nicht sein, dass sich die Kassen unisono zur integrierten Versorgung bekennen und andrerseits mit Pseudoprodukten dem Hausarztmodell das Wasser abgraben. Da die Versorgungsqualität in der Schweiz generell hoch sei, könne sich eine optimal koordinierte Hausarztmedizin nicht über die Qualität von einer ineffizienten Maximalme-

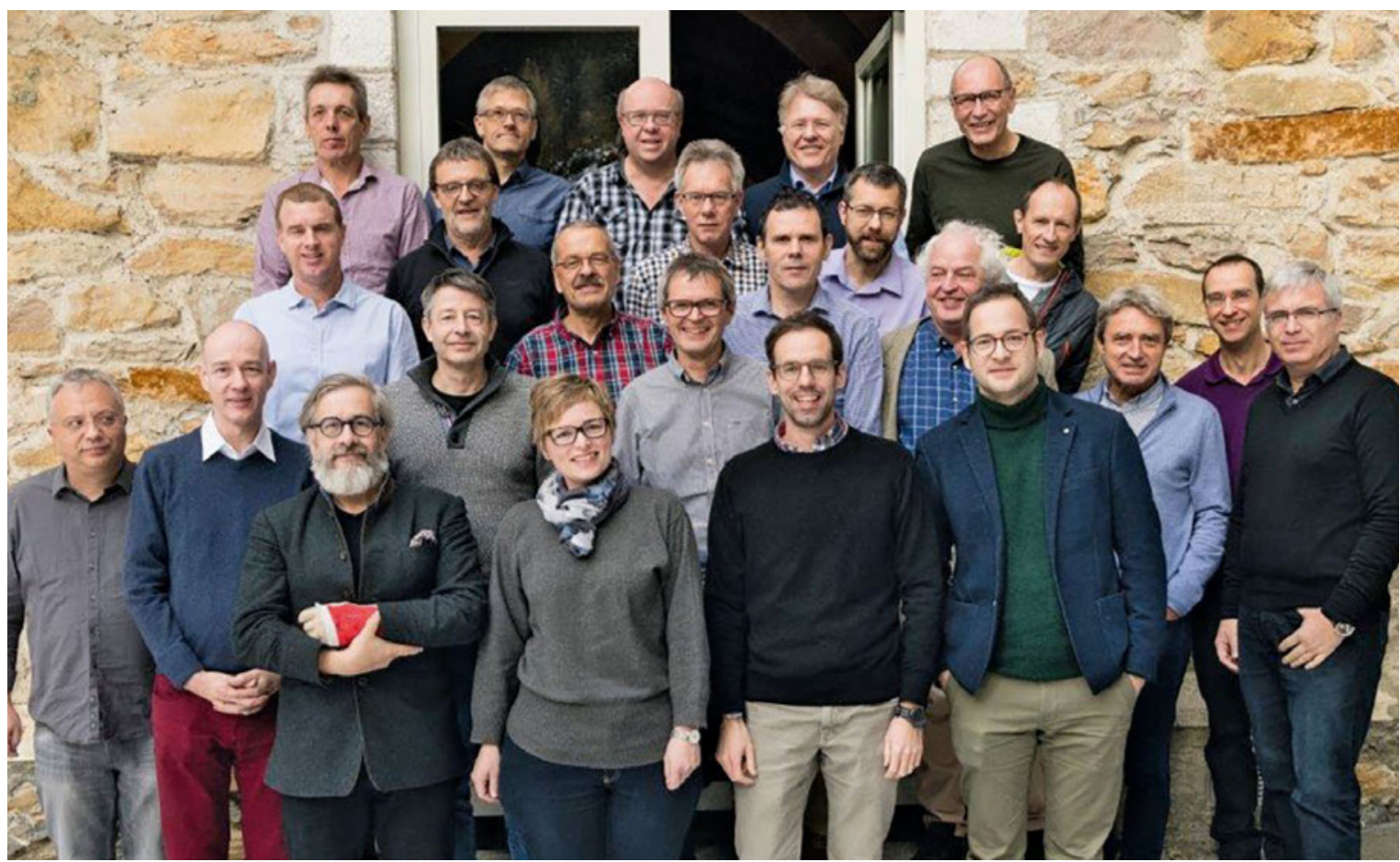

Führende Exponenten im Gesundheitswesen engagieren sich für eine starke integrierte Versorgung. Aufnahme vom Workshop in Scuol vom Dezember 2017 
dizin abgrenzen. Es sei deshalb unabdingbar, dass sich das Hausarztmodell über den besten Preis von allen anderen Produkten abgrenze. Die höhere Effizienz in den Hausarztmodellen sei wissenschaftlich bestens dokumentiert - die dadurch gesparten Kosten müssten deshalb zwingend in Form der günstigsten Prämie an die Patienten weitergegeben werden.

\section{Expertenbericht des BAG zur Eindämmung der Gesundheitskosten}

Wolfgang Czerwenka von Argomed stellte den kürzlich erschienenen Expertenbericht des BAG zur Eindämmung der Gesundheitskosten vor. Darin würden zwar eine Stärkung der koordinierten Versorgung und eine Förderung von Netzwerken vorgeschlagen. «Bedauerlicherweise wird das Hausarztmodell im Bericht mit keinem Wort spezifisch erwähnt, obwohl die vorgeschlagenen Massnahmen in vielen Punkten dem Konzept des Hausarztmodells entsprechen und wir seit 20 Jahren grosse Erfahrung in diesem Bereich haben.»

\section{Leistungsparameter müssen mit den Leistungs-} erbringern weiterentwickelt werden

Pius Zängerle, Direktor des Krankenkassenverbandes curafutura, ortete hier Potential für mehr Zusammenarbeit: Die MC-Bewegung und die Krankenversicherer hätten in der nutzenorientierten Medizin (outcomebzw. value-based) ein fundamentales gemeinsames Interesse. Dieses gelte es in Zukunft besser zu nutzen. Um das zu erreichen, brauche es auf mehreren Ebenen

\section{Der TARMED würde hier weiterhin als Basis- sprache fungieren, standardisierte Prozesse könnten jedoch in bilateralen Abmachungen mit den Dienstleistern pauschaliert werden.}

Anpassungen. Leistungsparameter (im Sinne von payfor-performance) müssten in kontinuierlicher Zusammenarbeit mit den Leistungserbringern, also den Ärzten - und nicht durch den Staat -, weiterentwickelt und angepasst werden. Eva Blozik von der Helsana ging in ihrem Referat näher auf den aktuellen Stand und die Anforderungen an Qualitätsindikatoren für die ergebnisorientierte Vergütung ein: «Wir können nicht alles messen, aber Qualität ist durchaus messbar.»

\section{Gemischtes Vergütungssystem}

Curafutura-Direktor Pius Zängerle stellte zudem die Idee eines gemischten Vergütungssystems vor. Der TARMED würde hier weiterhin als Basissprache fungieren, standardisierte Prozesse wie gewisse Aspekte in der Betreuung chronisch Kranker könnten jedoch in bilateralen Abmachungen mit den Dienstleistern pauschaliert werden. Damit solche Anpassungen möglich werden, erwarte er von ärztlicher Seite aber mehr Grösse und Vernetzung - ein kleines Netz genüge nicht.

\section{Allseitiges Bekenntnis für mehr integrierte Versorgung}

\section{Rekrutierung von Nachwuchs ist eine Heraus- forderung}

Einig war man sich bezüglich der Bedeutung einer hochstehenden, integrierten und interprofessionellen Versorgung, welche es weiterzuentwickeln gelte. Professor Oliver Senn vom Institut für Hausarztmedizin

\section{Viele Patienten würden im fragmentierten und komplexen Versorgungsdschungel alleine gelassen.}

zeigte den Bedarf an weiterer Forschung im Gebiet der Interprofessionalität und die Herausforderungen in der Rekrutierung von zukünftigen Hausärzten auf. Von Ärzteseite wurden zudem zahlreiche Projekte im Bereich der integrierten Versorgung vorgestellt. Als Beispiel sei hier das erfolgreiche PatientenschulungsProgramm von PizolCare genannt, welches von dessen Leiter Urs Keller präsentiert wurde.

\section{Es ginge auch einfacher - das Potential von Telemedizin und Co.}

Auch die Vertreter der Krankenkassen bekannten sich klar zur integrierten und kontinuierlichen Versorgung, wie sie die Hausarztmedizin seit langem praktiziert. Oliver Reich von sante24 stellte die enorme Dynamik im Bereich der virtuellen und telemedizinischen Dienstleistungen vor. "Wir wissen, dass das, was ihr tut, nicht falsch ist», meinte er zu den Vertretern der Hausarztnetze - «aber wir haben viel mehr Potential.» Viele Patienten würden im fragmentierten und komplexen Versorgungsdschungel alleine gelassen. Zudem gehe er davon aus, dass zu viele Patienten in klassischen Konsultationen gesehen würden, obwohl es auch einfacher ginge. Kaiser Permanente - eine integrierte Versorgungsorganisation in den USA - führe bereits $40 \%$ ihrer Arztkonsultationen virtuell durch Das Bedürfnis für mehr virtuelle und telemedizinische Dienstleistungen wurde auch von Seite der Ärztenetzte anerkannt. So betonte Felix Huber von mediX den Bedarf an Callcentern in Ergänzung und direkter Verbindung zum klassischen «hausärztlichen» $\mathrm{Zu}$ hause der Patienten. Andrerseits sei hier noch viel Bedarf an entsprechender Versorgungsforschung. Es 
müsse sichergestellt werden, dass nicht mehr Nachfrage ohne konkreten medizinischen Gegenwert geschaffen werde.

\section{Neben einer breiteren, für alle zugänglichen Austausch- und Informationsplattform soll künftig eine aktiv operierende Projektgruppe entstehen.}

Shop-in-Shop-Dienstleistungen in der Apotheke Auch seitens der Apotheker kam die Bereitschaft zur Teilnahme an der integrierten Versorgung klar zum Ausdruck. Christian Köpe vom Pharmazie-Logistikunternehmen Galenica stellte das Projekt «aprioris» vor, eine Shop-in-Shop-Dienstleistung durch Pflegeexperten in der Apotheke. «Hierbei handelt es sich um eine echte Verlagerung auf eine tiefere Kostenstufe mit gleichzeitigem Vorteil für die Patienten.» So könnten einfache Prozeduren wie Rachenabstriche, Otoskopien oder Wundversorgungen vorgenommen werden - der Patient bezahlt selbst, aber nur, wenn das Problem gelöst werden kann.

\section{Neuformation und Aufbruch in die Zukunft}

Um der Komplexität der geforderten Massnahmen beschlussfähig zu begegnen, bekannten sich die Teilnehmer abschliessend mit grosser Mehrheit zu einer Umgestaltung des aktuellen Workshop-Formats. Neben einer breiteren, für alle zugänglichen Austausch- und Informationsplattform soll künftig eine aktiv operierende Projektgruppe entstehen. Diese soll sich aus Vertretern aller interessierten Hausarztnetze und MC-Organisationen zusammensetzen und die Basis für die Weiterentwicklung von MC legen.
Aber auch systemische Reformschritte wurden hervorgehoben. Hier wurde wiederholt die fundamentale Bedeutung einer einheitlichen Finanzierung von ambulant und stationär (EFAS) erwähnt. «EFAS würde das Interesse der Krankenversicherer am Hausarztmodell stark erhöhen", meinte curafutura-Direktor Pius Zängerle. Da die Krankenversicherer an den stationären Leistungen aufgrund der kantonalen Subventionen aktuell weniger als die Hälfte bezahlen, fällt eine gute Betreuung von chronisch Kranken (bzw. die Verhinderung einer Hospitalisation) für sie wirtschaftlich weniger ins Gewicht. Umgekehrt würde eine einheitliche Finanzierung durch die Krankenversicherer einen Partner auf Seite der Dienstleister erfordern Managed Care ist hier die Antwort.

\section{Schlaglichter}

1. Die Hausarztmodelle mit Vertrag (ca. $25 \%$ der Bevölkerung) müssen bei allen Versicherungen die tiefsten Prämien haben EFAS ebnet den Weg.

2. Listenmodelle untergraben die Qualitätsarbeit der Ärztenetze und die Bemühungen derjenigen Krankenkassen, die für diese Qualitätsarbeit aufkommen. Infolge des negativen Bundesverwaltungsgerichtsentscheides müssen sich die Ärztenetze für eine KVG-Revision zur Unterbindung der Listung ohne Vertrag einsetzen, um damit das Ende der Listenmodelle einzuläuten.

3. Weiterentwicklung von Managed Care mit Callcentern, vertiefter Interprofessionalität und vorgelagerter integrierter Versorung (z.B. Apotheken)

4. Disease-Management mit Pauschalierung bleibt weiter auf der Traktandenliste.

5. Der MC-Workshop in Scuol wird durch eine aktiv operierende Projektgruppe und einen breiten Informationsanlass abgelöst Alle interessierten Hausarztnetze und MC-Organisationen sind eingeladen, sich zu beteiligen.

6. Die Hausarztmodelle und EFAS helfen sich gegenseitig: Mit EFAS sind zusätzlich ca. $5 \%$ Prämienrabatt möglich, ohne MC ist EFAS weit weniger wirksam. 